\title{
SÍNTESE DE NOVAS AMIDAS GRAXAS A PARTIR DA AMINÓLISE DE ÉSTERES METÍLICOS
}

Carolina R. Lopes, Caroline Da Ros Montes D’Oca, Rodrigo da C. Duarte, Márcia H. S. Kurz, Ednei G. Primel, Rosilene M. Clementin, Joaquín Ariel M. Villarreyes e Marcelo G. Montes D’Oca*

Escola de Química e Alimentos, Universidade Federal do Rio Grande, Av. Itália km 08 s/n, 96203-000 Rio Grande - RS, Brasil

Recebido em 11/11/09; aceito em 17/2/10; publicado na web em 21/5/10

\begin{abstract}
SYNTHESIS OF NEW FATTY ACIDS AMIDES FROM AMINOLYSIS OF FAMES. Recent biochemical and pharmacological studies have led to the characterization of different fatty acid amides as a new family of biologically active lipids. Here, we describe the synthesis of new amides from C16:0, 18:0, 18:1 and 18:1, OH fatty acids (FFA) families with cyclic and acyclic amines and demonstrate for the first time that these compounds produce citotoxic effects. Application of this method to the synthesis of fatty acid amides was performed using the esters aminolysis as a key step and various carboxylic amides were prepared in good yield from fatty acid methyl esters (FAMEs).
\end{abstract}

Keywords: anandamide; ricinoleic amides; FAMEs.

\section{INTRODUÇÃO}

Recentemente, estudos bioquímicos e farmacológicos vêm caracterizando as amidas graxas como uma nova família de lipídios biologicamente ativos..$^{1-6}$

Esta classe de compostos tem sido naturalmente encontrada em vegetais terrestres e aquáticos, em animais e micro-organismos. No reino vegetal, estão presentes nas algas Rizoclonium hierogliphicun, Zoostera marina e Sebastes mentella, nos vegetais Lepidium meyenii (maca) e Zanthoxylum sp..$^{7-11}$ No reino animal, as amidas graxas podem ser encontradas na esponja marinha Haliclona (Reniera) sp. e também em mamíferos como, por exemplo, em barbatanas de baleias, no fluído cérebro-espinhal de ratos e gatos, no plasma sanguíneo, no tecido adiposo, no cérebro, intestino e outros tecidos de ratos e porcos. ${ }^{12-23}$

A ocorrência de amidas graxas também foi verificada em organismos unicelulares como, por exemplo, a Corynebacterium nitrilophilus, algumas espécies de Streptomyces e células de câncer de mama. Na cianobactéria marinha Lyngbya majuscula foram identificadas as amidas graxas, hermitamidas, grenadamida, semiplenamidas $\mathrm{C}$ e as besar-hanamidas A e B. ${ }^{24-30}$

O estudo das amidas graxas teve início na década de 1950, quando foi descoberta a atividade anti-inflamatória de algumas frações do óleo de amendoim, da lecitina de soja e de gemas de ovos. O princípio ativo responsável por essas ações foi atribuído à palmitoiletanolamida (1) a primeira amida graxa isolada (Figura 1). ${ }^{31}$

A identificação das amidas graxas, a $N$-araquidonoiletanolamida, também chamada de anandamida (AEA, 2) ${ }^{32}$ e a cis-oleamida (cOA, 3), ${ }^{33}$ membros mais conhecidos e estudados desta grande família, abriu caminhos para a investigação das propriedades farmacológicas, identificação, síntese e isolamento de novas amidas graxas como, por exemplo, oleiletanolamida (4) e $\mathrm{N}$-benzil-15-Z-tetracosenamida (5, macamida) (Figura 1)..$^{10,34}$

O objetivo deste trabalho foi sintetizar novas amidas graxas derivadas de diferentes aminas a partir da reação de aminólise de ésteres graxos saturados e insaturados. Dentro deste contexto, também foi utilizado óleo de mamona para a síntese de ricinoleil amidas graxas.

*e-mail: dqmdoca@furg.br

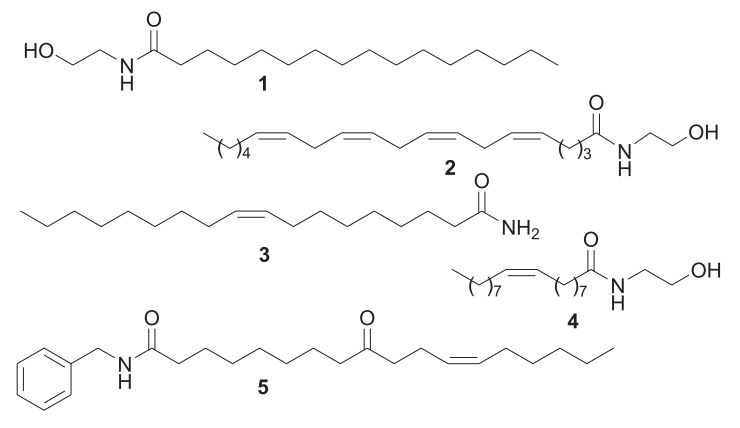

Figura 1. Amidas graxas biologicamente ativas, palmitoiletanolamida (1), anandamida (2), cis-oleamida (3), oleiletanolamida (4) e N-benzil-Ztetracosenamida (5)

\section{RESULTADOS E DISCUSSÃO}

De acordo com a literatura, a aminólise é um processo importante para a síntese de amidas e muitos métodos são descritos, tanto químicos quanto enzimáticos, para promover esta reação. ${ }^{35,36}$ No caso da síntese de amidas graxas envolvendo o processo de aminólise, a baixa reatividade do ácido graxo frente a amina fica ainda mais evidente. Por este motivo, poucos trabalhos tratam da síntese destas amidas e alternativas para solucionar este problema podem ser converter o ácido carboxílico em intermediários mais reativos, como haleto de acila, anidrido ou ésteres. ${ }^{37,38}$ Outras metodologias propõem o uso do anidrido do ácido propilfosfônico (PPAA) ${ }^{6,39}$ ou de carbodi-imidas ${ }^{40}$ para aumentar a reatividade de ácidos carboxílicos graxos e promover a síntese de amidas em um menor tempo de reação e temperatura. Khan e colaboradores, ${ }^{10}$ por exemplo, utilizaram DCC e DMAP na síntese da $N$-benzil-15-Z-tetracosenamida, amida graxa encontrada no vegetal Lepidium meyenii (maca).

Neste trabalho, descreveu-se a síntese de novas amidas graxas com base na similaridade estrutural das amidas já descritas na literatura como, por exemplo, a palmitoiletanolamida (1) ${ }^{41}$ anandamida (2), ${ }^{42}$ cis-oleamida (3) ${ }^{43}$ oleiletanolamida $(4)^{44} \mathrm{e}$ macamida $(5)^{10}$ (Figura 1$)$. A inovação ocorreu devido à presença dos diferentes substituintes ligados ao átomo de nitrogênio e a sua combinação com as cadeias graxas provenientes dos ácidos graxos comerciais, palmítico (6a), esteárico (6b) e oleico (6c) e do óleo de mamona (ricinus communis). ${ }^{45,46}$ 
Os ácidos graxos 6a-c (Esquema 1) foram esterificados utilizando-se $\mathrm{H}_{2} \mathrm{SO}_{4}$ e metanol a temperatura de $65{ }^{\circ} \mathrm{C}$ por $4 \mathrm{~h}$, formando os respectivos ésteres metílicos graxos $\mathbf{7 a - c .}$

O ricinoleato de metila (7d) foi obtido a partir da transesterificação da ricinoleína $(\mathbf{8})$ presente no óleo de mamona na presença de $\mathrm{NaOH}$ e metanol, sob aquecimento de $60^{\circ} \mathrm{C}$ por $1 \mathrm{~h}$ (Esquema 1).

Todos os ésteres foram isolados em bons rendimentos após purificação em coluna cromatográfica utilizando-se sílica gel e gradientes de hexano e acetato de etila (Tabela 1). Os compostos 7a-d foram caracterizados, tendo seus tempos de retenção comparados por GCFID com padrões cromatográficos comerciais.

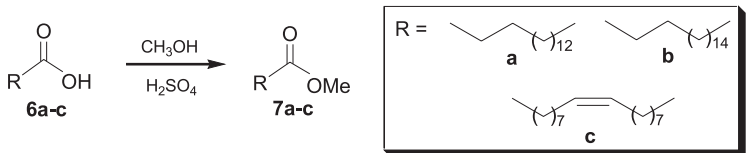

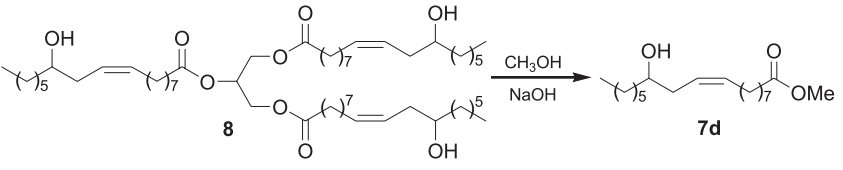

Esquema 1. Síntese dos ésteres metílicos 7 a-c e $7 \boldsymbol{d}$ a partir da esterificação de ácidos graxos e da transesterificação do óleo de mamona, respectivamente

Tabela 1. Síntese dos ésteres graxos 7a-d

\begin{tabular}{|c|c|c|c|c|}
\hline Entrada & $\begin{array}{c}\text { Material de } \\
\text { partida }\end{array}$ & $\begin{array}{l}\text { Condições } \\
\text { reacionais }\end{array}$ & $\begin{array}{c}\text { Éster graxo, } \\
\%\end{array}$ & $\begin{array}{l}\mathrm{IV}, \delta \mathrm{cm}^{-1} \\
\text { (ident.) }\end{array}$ \\
\hline 1 & 6a, C16:0 & $\begin{array}{l}\mathrm{CH}_{3} \mathrm{OH}, 1,5 \% \text { de } \\
\mathrm{H}_{2} \mathrm{SO}_{4,} 65^{\circ} \mathrm{C}, 4 \mathrm{~h}\end{array}$ & $7 \mathbf{a}, 83 \%$ & $\begin{array}{l}1739(\mathrm{C}=\mathrm{O}) \\
1172(\mathrm{C}-\mathrm{O})\end{array}$ \\
\hline 2 & 6b, C18:0 & $\begin{array}{l}\mathrm{CH}_{3} \mathrm{OH}, 1,5 \% \text { de } \\
\mathrm{H}_{2} \mathrm{SO}_{4}, 65^{\circ} \mathrm{C}, 4 \mathrm{~h}\end{array}$ & $7 b, 94 \%$ & $\begin{array}{l}1743(\mathrm{C}=\mathrm{O}) \\
1170(\mathrm{C}-\mathrm{O})\end{array}$ \\
\hline 3 & 6c, C18:1 & $\begin{array}{l}\mathrm{CH}_{3} \mathrm{OH}, 1,5 \% \text { de } \\
\mathrm{H}_{2} \mathrm{SO}_{4}, 65^{\circ} \mathrm{C}, 4 \mathrm{~h}\end{array}$ & 7c, $85 \%$ & $\begin{array}{l}1743(\mathrm{C}=\mathrm{O}) \\
1463(\mathrm{C}=\mathrm{C}) \\
1170(\mathrm{C}-\mathrm{O})\end{array}$ \\
\hline 4 & $\begin{array}{l}\mathbf{8}, \text { óleo de } \\
\text { mamona }\end{array}$ & $\begin{array}{l}\mathrm{CH}_{3} \mathrm{OH}, 1,5 \% \text { de } \\
\mathrm{NaOH} 60^{\circ} \mathrm{C}, 1 \mathrm{~h}\end{array}$ & $7 d, 90 \%$ & $\begin{array}{l}3414(\mathrm{O}-\mathrm{H}) \\
1731(\mathrm{C}=\mathrm{O}) \\
1459(\mathrm{C}=\mathrm{C}) \\
1178(\mathrm{C}-\mathrm{O})\end{array}$ \\
\hline
\end{tabular}

Os ésteres metílicos graxos 7a-d foram submetidos à reação de aminólise na presença de pirrolidina, piperidina, morfolina, etanolamina ou benzilamina para a síntese das amidas graxas 9-13a-d (Esquema 2). As condições reacionais e os rendimentos são mostrados na Tabela 2.

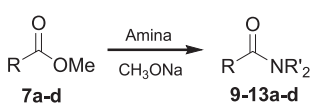

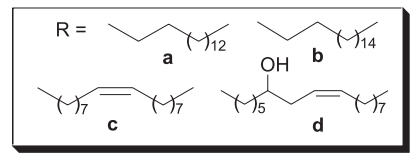

Esquema 2. Síntese das amidas graxas 9-13a-d a partir da aminólise de ésteres graxos, 49 .

Na maioria dos casos as amidas graxas foram obtidas em bons rendimentos utilizando uma proporção molar de 1:6, éster graxo:amina. Entretanto, devido aos baixos rendimentos observados com o uso das aminas cíclicas de 6 membros, piperidina e morfolina (Tabela 2, entradas 5-12), investigamos o procedimento descrito por $\mathrm{Ahmad}^{47}$ para a síntese de dietanolamidas graxas.

O metóxido de sódio, sintetizado a partir de metanol seco e sódio metálico, foi dissolvido na amina e após adicionado o éster metílico ao meio reacional para obtenção das amidas graxas 10,11a-d. De acordo com os resultados obtidos (Tabela 2, entradas 13-20), a utilização do metóxido de sódio proporcionou o aumento da conversão dos ésteres nas amidas graxas, o emprego de menor quantidade estequiométrica da amina e a diminuição da temperatura usada na reação.

Após a caracterização dos compostos, foi observada uma grande semelhança nos dados espectroscópicos para as amidas 9-13a e 9-13b derivadas dos ácidos palmítico e esteárico, respectivamente. Entretanto, após analise por CG-EM foram observados nos espectros de massas da palmitoilbenzilamida graxa (13a) e da estearilbenzilamida graxa (13b), por exemplo, os íons moleculares de $m / z, 345$ e 373, respectivamente. Em ambos os casos, também foram observados os fragmentos provenientes do rearranjo de McLafferty $(\mathrm{m} / \mathrm{z}, 149)$ e da $\beta$-clivagem $(\mathrm{m} / \mathrm{z}, 162)$.

\section{CONCLUSÃO}

De acordo com os resultados obtidos, as amidas graxas 9-13a-d foram produzidas em bons rendimentos utilizando como etapa chave a aminólise de ésteres graxos. Cabe salientar que, através deste procedimento, foram sintetizadas amidas graxas derivadas de aminas cíclicas e acíclicas e, também, amidas derivadas da ricinoleína principal componente do óleo de mamona, todas inéditas na literatura.

Os ensaios de atividade biológica dos compostos 9-13a-d encontramse em andamento. Até o momento observou-se que as pirrolidilamidas graxas 9a-d foram citotóxicas em células eritroleucêmicas humanas do tipo K562 quando ministradas em doses terapêuticas sendo que, para a palmitoilpirrolidil amida (9a) foi verificada a maior atividade..$^{48,49}$ Os estudos terão prosseguimento visando investigar a relação estrutura-atividade das amidas graxas sintetizadas, bem como o seu efeito em leucócitos saudáveis e em linhagens eritroleucêmicas resistentes à quimioterapia.

\section{PARTE EXPERIMENTAL}

\section{Reagentes e equipamentos}

Todos os reagentes utilizados neste trabalho foram obtidos de fontes comerciais e, quando necessário, purificados previamente por destilação. O óleo de mamona foi obtido da Delaware Ltda, Porto Alegre, RS, Brasil (Características: IA $=0,80 \mathrm{mg} \mathrm{g}^{-1}$; IS=179,9 mg $\mathrm{g}^{-1} ; \mathrm{II}=83,50 \mathrm{cg} \mathrm{g}^{-1} ; \mathrm{IH}=160,0 \mathrm{mg} \mathrm{g}^{-1}$ e d=0,96 $\left.\mathrm{g} \mathrm{mL}^{-1}\right)$. Os padrões cromatográficos de ácidos graxos foram obtidos da Sigma-Aldrich Chemicals. As temperaturas de fusão foram determinadas em um equipamento Fisatom 430D, utilizando-se método capilar.

Os espectros de RMN foram obtidos em espectrômetro Bruker de $250 \mathrm{MHz}$ e Varian VNMRs de $300 \mathrm{MHz}$. As amostras foram dissolvidas em clorofórmio deuterado $\left(\mathrm{CDCl}_{3}\right)$. Tetrametilsilano (TMS) foi utilizado como padrão de referência interna. Os espectros na região do infravermelho foram obtidos em espectrofotômetro Shimadzu-IR Prestige-21, com leituras na região de 4000 a $400 \mathrm{~cm}^{-1}$.

As análises cromatográficas dos ésteres e das amidas graxas foram efetuadas em um cromatógrafo a gás Shimadzu QP2010 equipado com injetor split/splitless, detector por ionização em chama (DIC) e coluna capilar RTX-WAX ( $30 \mathrm{~m} \times 0,25 \mathrm{~mm}, 0,25 \mu \mathrm{m}$ de polietilenoglicol) e tratamento dos dados através do software GC Solution. Foram utilizadas as seguintes condições: gás carreador, hidrogênio, vazão de $1 \mathrm{~mL} / \mathrm{min}$, split 1:100, volume de amostra de $1 \mu \mathrm{L}$, temperatura programada do forno: temperatura inicial $80^{\circ} \mathrm{C}$, mantida por 1 min, a seguir rampa de $10{ }^{\circ} \mathrm{C} / \mathrm{min}$ até $180^{\circ} \mathrm{C}$ e, a seguir, rampa de 7 ${ }^{\circ} \mathrm{C} / \mathrm{min}$ até $240{ }^{\circ} \mathrm{C}$, com tempo total de análise de $30 \mathrm{~min}$, temperatura do injetor de $250{ }^{\circ} \mathrm{C}$ e temperatura do detector de $260{ }^{\circ} \mathrm{C}$.

As análises por CG-EM foram realizadas em um cromatógrafo a gás Shimadzu GC 2010 acoplado ao espectrômetro de massas - QP2010 Plus, com injetor automático AOC-20i, coluna capilar RTX-5MS (5\% 
Tabela 2. Síntese das amidas graxas 9-13a-d a partir da aminólise de ésteres graxos

\begin{tabular}{|c|c|c|c|c|c|c|}
\hline Entrada & Éster & Amina & $\begin{array}{c}\text { Éster:amina: } \\
\mathrm{CH}_{3} \mathrm{ONa} \\
(\mathrm{mols})\end{array}$ & $\mathrm{T},{ }^{\circ} \mathrm{C}$ & Amida graxa, $\%$ & \\
\hline 1 & $7 a$ & pirrolidina & 1:6:0 & 90 & & $\begin{array}{l}9 a \\
60 \%\end{array}$ \\
\hline 2 & $7 b$ & pirrolidina & 1:6:0 & 90 & & $\begin{array}{l}\mathbf{9 b}, \\
67 \%\end{array}$ \\
\hline 3 & $7 c$ & pirrolidina & 1:6:0 & 90 & & $\begin{array}{c}9 c, \\
66 \%\end{array}$ \\
\hline 4 & $7 d$ & pirrolidina & $1: 6: 0$ & 90 & & $\begin{array}{l}9 \mathbf{9}, \\
78 \%\end{array}$ \\
\hline 5 & $7 \mathbf{a}$ & piperidina & $1: 6: 0$ & 90 & & $\begin{array}{l}10 a \\
6 \%\end{array}$ \\
\hline 6 & $7 b$ & piperidina & 1:6:0 & 90 & & $\begin{array}{l}\text { 10b, } \\
\text { n.r. }\end{array}$ \\
\hline 7 & $7 c$ & piperidina & 1:6:0 & 90 & & $\begin{array}{l}10 \mathrm{c}, \\
10 \%\end{array}$ \\
\hline 8 & $7 d$ & piperidina & 1:6:0 & 90 & & \\
\hline 9 & $7 \mathbf{a}$ & morfolina & 1:6:0 & 130 & & $\begin{array}{l}\text { 11a, } \\
30 \%\end{array}$ \\
\hline 10 & $7 b$ & morfolina & $1: 6: 0$ & 130 & & $11 \mathrm{~b}$, \\
\hline 11 & $7 c$ & morfolina & 1:6:0 & 130 & & $11 \mathrm{c}$, \\
\hline 12 & $7 d$ & morfolina & 1:6:0 & 130 & & \\
\hline 13 & $7 a$ & piperidina & $1: 3: 1$ & 75 & & $\begin{array}{l}\text { 10a, } \\
75 \%\end{array}$ \\
\hline 14 & $7 b$ & piperidina & $1: 3: 1$ & 75 & & 10b, \\
\hline 15 & $7 c$ & piperidina & $1: 3: 1$ & 75 & & 10c, \\
\hline 16 & $7 d$ & piperidina & $1: 3: 1$ & 75 & & \\
\hline 17 & $7 \mathbf{a}$ & morfolina & $1: 3: 1$ & 75 & & 11a, \\
\hline
\end{tabular}

Tabela 2. Continuação

\begin{tabular}{lccccc}
\hline Entrada Éster & Amina & $\begin{array}{c}\text { Éster:amina: } \\
\mathrm{CH}_{3} \mathrm{ONa} \\
(\mathrm{mols})\end{array}$ & $\mathrm{T},{ }^{\circ} \mathrm{C}$ & Amida graxa, \% & \\
\hline 18 & $\mathbf{7 b}$ & morfolina & $1: 3: 1$ & 75 &
\end{tabular}

$19 \quad$ 7c morfolina $1: 3: 1 \quad 75$
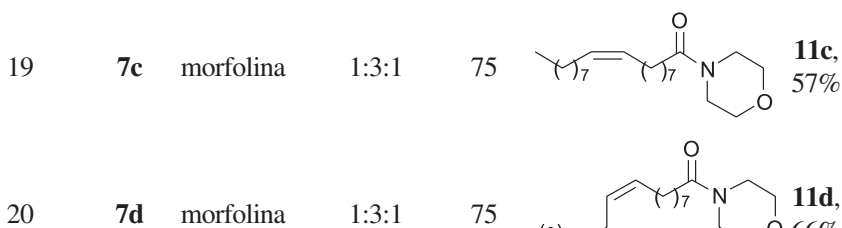

7d morfolina

$1: 3: 1$

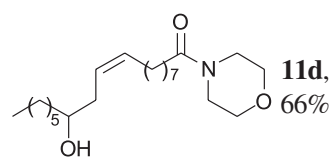

7a etanolamina

$1: 6: 0$

etanolamina $1: 6: 0$

$22 \quad 7 \mathbf{b}$ etanolamina $1: 6: 0 \quad 130$

30

${ }_{\mathrm{H}} \mathrm{H}_{\mathrm{H}}^{\mathrm{O}} \sim \mathrm{OH}$

$12 \mathrm{a}$, $62 \%$

$23 \quad 7 \mathrm{c}$ etanolamina $1: 6: 0$

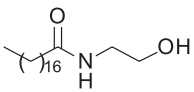

12b, $88 \%$

$130 \mathrm{H}_{7}=\mathrm{M}_{7} \mathrm{~N}_{\mathrm{H}} \sim \mathrm{OH}_{66 \%}$

24

7d etanolamin

1:6:0

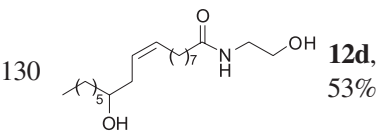

25

7a benzilamina

1:6:0
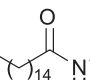

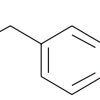

13a $65 \%$

26

7b benzilamina

$1: 6: 0$<smiles>CCC(=O)NCc1ccccc1</smiles>

13b, $60 \%$

7c benzilamina

1:6:0<smiles>CC=CCC(=O)NCc1ccccc1</smiles>

28

7d

d ben

$1: 6 \cdot 0$

fenil e $95 \%$ metilpolissiloxano, $30 \mathrm{~m} \times 0,25 \mathrm{~mm}, 0,25 \mu \mathrm{m}$ ) e tratamento dos dados através do software GC-MS Solution. As condições de análise foram: gás carreador: hélio, vazão de $1,0 \mathrm{~mL} / \mathrm{min}$, injeção splitless, volume de $1 \mu \mathrm{L}$, temperatura programada do forno: temperatura inicial de $150{ }^{\circ} \mathrm{C}$ mantida por $3 \mathrm{~min}$, a seguir rampa de $15^{\circ} \mathrm{C} / \mathrm{min}$ até $300^{\circ} \mathrm{C}$, mantida por $17 \mathrm{~min}$, com tempo total de análise de $30 \mathrm{~min}$, temperatura do injetor de $250{ }^{\circ} \mathrm{C}$, temperatura da fonte de $250{ }^{\circ} \mathrm{C}$, temperatura da interface de $280^{\circ} \mathrm{C}$, voltagem de ionização $70 \mathrm{eV}$, corrente de ionização $150 \mu \mathrm{A}$; modo de varredura de $50-400 \mathrm{~m} / \mathrm{z}$.

\section{Sínteses}

Sintese dos ésteres graxos $7 a-c$

Em um balão contendo o ácido graxo 6a-c (25 mmols) adicionouse $15 \mathrm{~mL}$ de solução metanólica contendo $1,5 \%$ em massa de $\mathrm{H}_{2} \mathrm{SO}_{4}$ em relação ao ácido graxo, mantendo-se à temperatura constante de 60 ${ }^{\circ} \mathrm{C}$ e agitação magnética por $4 \mathrm{~h}$. Após, lavou-se com água destilada, adicionou-se sulfato de magnésio $\left(\mathrm{MgSO}_{4}\right)$ filtrou-se e evaporou-se o solvente. A reação foi monitorada por cromatografia de camada delgada (CCD) utilizando como eluente hexano:acetato de etila, 9:1. A purificação dos ésteres graxos $\mathbf{7 a - c}$ foi realizada através de coluna cromatográfica utilizando sílica gel. 


\section{Síntese dos ésteres graxos $7 d$}

Em um balão contendo óleo de mamona (50 mmols, 47,17 g) adicionaram-se $12 \mathrm{~mL}$ de solução metanólica contendo $1 \%$ em massa de $\mathrm{NaOH}$ em relação ao óleo, deixando-se à temperatura constante de $60{ }^{\circ} \mathrm{C}$ e agitação por $1 \mathrm{~h}$. Após, lavou-se com água destilada, adicionou-se sulfato de magnésio $\left(\mathrm{MgSO}_{4}\right)$ filtrou-se e evaporou-se o solvente. A reação foi monitorada por cromatografia de camada delgada (CCD) utilizando como eluente hexano:acetato de etila, 7:3. A purificação do éster graxo $7 \mathbf{d}$ foi realizada através de coluna cromatográfica utilizando sílica gel.

A massa molecular do óleo de mamona foi obtida através das massas moleculares dos triglicerídeos (ricinoleína, 944,952 g, estearina 890,906 g, oleína 896,954 g e linoleína, 903,002 g) ponderadas com a composição molar obtida a partir do perfil graxo do óleo, 92,2; 3,99; 3,01 e 0,80\% dos ácidos ricinoleico, esteárico, oleico e linoleico, respectivamente, determinado por cromatografia gasosa.

$$
\begin{gathered}
\mathrm{M}_{\text {oleo }}=\frac{\mathbf{1}}{\Sigma \frac{z}{\mathrm{M}_{\mathrm{TG}}}} \\
\mathrm{M}_{\text {oleo }}=\frac{1}{\frac{0,922}{944,952}+\frac{0,0080}{890,906}+\frac{0,0301}{896,954}+\frac{0,0399}{903,002}}=943,396 \frac{\mathrm{g}}{\mathrm{mol}}
\end{gathered}
$$

onde: $\mathrm{z}$ = fração mássica $(\mathrm{p} / \mathrm{p}) ; \mathrm{M}=$ massa molecular do TG $(\mathrm{g} / \mathrm{mol})$.

\section{Palmitato de metila $(7 a)^{50}$}

Rendimento: $83 \%$. Aspecto: sólido branco, p.f. $30-32{ }^{\circ} \mathrm{C}$. CCD: Rf = 0,9; AcOEt:Hex (1:1). IV ( NaCl, filme): $\mathrm{cm}^{-1}$ (intensidade) 2916 (F); 2848 (F); 1739 (F); 1462 (f); 1435 (f); 1172 (f); 883 (f).

\section{Estearato de metila $(\mathbf{7 b})^{50,51}$}

Rendimento: $94 \%$. Aspecto: sólido branco, p.f.: $38-40{ }^{\circ} \mathrm{C}$. CCD: Rf = 0,9; AcOEt:Hex (1:1). IV ( $\mathrm{NaCl}$, filme): $\mathrm{cm}^{-1}$ (intensidade) 2922 (F); 2852 (F); 1743 (F); 1463 (m); 1435 (m); 1359 (f); 1195 (f); $1170(\mathrm{~m})$.

\section{Oleato de metila $(7 c)^{50}$}

Rendimento: $85 \%$. Aspecto: líquido amarelo, CCD: $\mathrm{Rf}=0,9$; AcOEt:Hex (1:1). IV (NaCl, filme): $\mathrm{cm}^{-1}$ (intensidade) 2926 (F); 2854 (F); 1743 (F); 1463 (m); 1436 (m); 1197 (f); 1170 (f).

\section{Ricinoleato de metila $(\mathbf{7 d})^{52,53}$}

Rendimento: $90 \%$. Aspecto: líquido amarelo, CCD: $\mathrm{Rf}=0,8$; AcOEt:Hex (1:1). IV (NaCl, filme): $\mathrm{cm}^{-1}$ (intensidade) $3414(\mathrm{~F})$, 2924 (F); 2856 (F), 1731 (m); 1712 (f), 1459 (f), 1241 (f), 1029 (f), 848 (f), 720 (f). RMN ${ }^{1} \mathrm{H}\left(300 \mathrm{MHz}, \mathrm{CDCl}_{3}\right) \delta 5,32-5,65$ (m, 2H); $3,66(\mathrm{~s}, 3 \mathrm{H}) ; 3,61-3,72(\mathrm{~m}, 1 \mathrm{H}) ; 2,30(\mathrm{t}, J=7,4 \mathrm{~Hz}, 2 \mathrm{H}) ; 2,21$ (t, $J$ $=7,2 \mathrm{~Hz}, 2 \mathrm{H}) ; 2,03-2,11(\mathrm{~m}, 2 \mathrm{H}) ; 1,30-1,71(\mathrm{~m}, 21 \mathrm{H}) ; 0,90(\mathrm{t}, J=$ $6,8 \mathrm{~Hz}, 3 \mathrm{H}) . \mathrm{RMN}{ }^{13} \mathrm{C}\left(75 \mathrm{MHz}, \mathrm{CDCl}_{3}\right) \delta 174 ; 133 ; 125 ; 72 ; 60$; $36 ; 35 ; 34 ; 32$ (2C); 29 (5C); 27; 25 (2C); 22; 14.

\section{Sintese de amidas graxas 9-13a-d}

\section{Aminólise de ésteres graxos}

Em um balão adicionou-se o éster metílico $7 \mathbf{a}-\mathbf{d}$ ( 2 mmols $)$ e a amina (12 mmols) nas temperaturas de 90 ou $130{ }^{\circ} \mathrm{C}$, deixandose sob agitação constante durante $24 \mathrm{~h}$. A reação foi monitorada por cromatografia de camada delgada (CCD), tendo como eluente hexano:acetato de etila, 7:3 e clorofórmio:metanol, 97:3 para os derivados da etanolamina. Os produtos 9-13a-d foram purificados através de coluna cromatográfica utilizando sílica gel.
Aminólise de ésteres graxos na presença de metóxido de sódio

O metóxido de sódio foi preparado a partir de metanol seco (44 $\mathrm{mL})$ e sódio metálico $(0,22 \mathrm{~mol}, 5 \mathrm{~g})$ à temperatura ambiente sob agitação por $30 \mathrm{~min}$. Após, evaporou-se o metanol com o auxílio de evaporador rotatório. O produto foi mantido em local seco e protegido da umidade.

Em um balão adicionou-se metóxido de sódio (2 mmols, $108 \mathrm{mg}$ ) e a respectiva amina (6 mmols) a temperatura ambiente, deixando-se sob agitação constante por $0,5 \mathrm{~h}$. Após, elevou-se a temperatura até $75{ }^{\circ} \mathrm{C}$ e adicionou-se lentamente o éster metílico $7 \mathbf{a}-\mathbf{d}$ ( $\left.2 \mathrm{mmols}\right)$ deixando-se sob agitação magnética por $24 \mathrm{~h}$. A reação foi acompanhada por cromatografia de camada delgada (CCD), tendo como eluente hexano:acetato de etila, 7:3. Os produtos 10a-d e 11a-d foram purificados através de coluna cromatográfica utilizando sílica gel.

\section{N-Pirrolidinil-hexadecanamida $(\mathbf{9 a})$}

$\mathrm{C}_{20} \mathrm{H}_{39}$ NO. Rendimento: $60 \%$. Aspecto: sólido branco, p.f.: 44-45 ${ }^{\circ} \mathrm{C}$. CCD: Rf = 0,5; AcOEt:Hex (1:1). IV ( $\mathrm{NaCl}$, filme): $\mathrm{cm}^{-1}$ (intensidade) 2974 (f); 2955 (f); 2917 (F); 2850 (F); 1636 (F); 1462 (m); 1325 (f); 1164 (f); 863 (f); 718 (f); 659 (f). $\mathrm{RMN}^{1} \mathrm{H}\left(250 \mathrm{MHz}, \mathrm{CDCl}_{3}\right) \delta$ $3,42(\mathrm{~m}, 4 \mathrm{H}) ; 2,23(\mathrm{t}, J=7,5 \mathrm{~Hz}, 2 \mathrm{H}) ; 1,93(\mathrm{~m}, 4 \mathrm{H}) ; 1,62(\mathrm{~m}, 2 \mathrm{H})$; $1,23(\mathrm{~m}, 24 \mathrm{H}) ; 0,85(\mathrm{t}, J=6,5 \mathrm{~Hz}, 3 \mathrm{H}) . \mathrm{RMN}^{13} \mathrm{C}\left(62,5 \mathrm{MHz}, \mathrm{CDCl}_{3}\right)$ $\delta 171,85 ; 46,58(2 \mathrm{C}) ; 45,53 ; 34,85 ; 31,90(2 \mathrm{C}) ; 29,94(2 \mathrm{C}) ; 29,66$ (2C); 29,53; 29,51; 29,46; 29,34; 26,12; 24,90; 24,40; 22,66; 14,09 .

\section{N-Pirrolidinil-octadecanamida $(\mathbf{9 b})$}

$\mathrm{C}_{22} \mathrm{H}_{43}$ NO. Rendimento: $67 \%$. Aspecto: sólido branco, p.f.: 38-39 ${ }^{\circ} \mathrm{C}$. CCD: Rf = 0,5; AcOEt:Hex (1:1). IV ( NaCl, filme): $\mathrm{cm}^{-1}$ (intensidade) 2954 (f); 2917 (F); 2849 (F); 1637 (F); 1462 (f); 1441 (m); 1331 (f); 1250 (f); 1168 (f); 859 (f); 722 (f). RMN ${ }^{1} \mathrm{H}(250 \mathrm{MHz}$, $\left.\mathrm{CDCl}_{3}\right) \delta 3,42(\mathrm{~m}, 4 \mathrm{H}) ; 2,23(\mathrm{t}, J=7,5 \mathrm{~Hz}, 2 \mathrm{H}) ; 1,85(\mathrm{~m}, 4 \mathrm{H}) ; 1,62$ $(\mathrm{m}, 2 \mathrm{H}) ; 1,24(\mathrm{~m}, 28 \mathrm{H}) ; 0,86(\mathrm{t}, J=6,7 \mathrm{~Hz}, 3 \mathrm{H}) . \mathrm{RMN}{ }^{13} \mathrm{C}(62,5$ $\left.\mathrm{MHz}, \mathrm{CDCl}_{3}\right) \delta 171,84 ; 46,58(2 \mathrm{C}) ; 45,53 ; 34,85 ; 31,90$ (2C); 29,67 (6C); 29,54; 29,52; 29,46; 29,34; 26,12; 24,95; 24,40; 22,67; 14,09.

\section{N-pirrolidinil-9-Z-octadecenamida $(\mathbf{9 c})$}

$\mathrm{C}_{22} \mathrm{H}_{41} \mathrm{NO}$. Rendimento: $66 \%$. Aspecto: líquido amarelo-palha. CCD: Rf = 0,5; AcOEt:Hex (1:1). IV ( $\mathrm{NaCl}$, filme): $\mathrm{cm}^{-1}$ (intensidade) 2923 (F); 2853 (F); 1647 (F); 1428 (m); 1337 (f); 1247 (f); 1223 (f); 1192 (f); 1168 (f); 718 (f). RMN ${ }^{1} \mathrm{H}\left(250 \mathrm{MHz}, \mathrm{CDCl}_{3}\right) \delta 5,32$ (m, $2 \mathrm{H}) ; 3,42(\mathrm{~m}, 4 \mathrm{H}) ; 2,23(\mathrm{t}, J=7,5 \mathrm{~Hz}, 2 \mathrm{H}) ; 2,00-1,79(\mathrm{~m}, 8 \mathrm{H}) ; 1,63$ $(\mathrm{m}, 2 \mathrm{H}) ; 1,30(\mathrm{~m}, 20 \mathrm{H}) 0,89(\mathrm{t}, J=6,5 \mathrm{~Hz}, 3 \mathrm{H}) . \mathrm{RMN}^{13} \mathrm{C}(62,5 \mathrm{MHz}$, $\left.\mathrm{CDCl}_{3}\right) \delta 171,84 ; 129,92 ; 129,78 ; 46,59 ; 45,55 ; 34,84 ; 32,58 ; 31,88$; 31,$76 ; 29,75 ; 29,71 ; 29,67 ; 29,50 ; 29,35 ; 29,15 ; 28,96 ; 27,19 ; 27,17$; 26,$12 ; 24,92 ; 24,40 ; 22,66 ; 14,09$.

\section{N-Pirrolidinil-12-hidróxi-9-Z-octadecenamida (9d)}

$\mathrm{C}_{22} \mathrm{H}_{41} \mathrm{NO}_{2}$. Rendimento: $78 \%$. Aspecto: líquido amarelo-palha. CCD: Rf = 0,3; AcOEt:Hex (1:1). IV ( $\mathrm{NaCl}$, filme): $\mathrm{cm}^{-1}$ (intensidade) $3412(\mathrm{~F}) ; 2926(\mathrm{~F}) ; 2856(\mathrm{~F}) ; 1627(\mathrm{~F}) ; 1448(\mathrm{~F}) ; 1342(\mathrm{~m})$; 1226 (m); 1041 (f); 860 (f); 752 (f). RMN ${ }^{1} \mathrm{H}\left(300 \mathrm{MHz}, \mathrm{CDCl}_{3}\right) \delta$ $5,51(\mathrm{~m}, 1 \mathrm{H}) ; 5,42(\mathrm{~m}, 1 \mathrm{H}) ; 3,68(\mathrm{~m}, 1 \mathrm{H}) ; 3,43(\mathrm{~m}, 4 \mathrm{H}) ; 2,27$ (sl, $1 \mathrm{H}) ; 2,25(\mathrm{~m}, 4 \mathrm{H}) ; 2,03(\mathrm{~m}, 2 \mathrm{H}) ; 1,95(\mathrm{~m}, 2 \mathrm{H}) ; 1,85(\mathrm{~m}, 2 \mathrm{H}) ; 1,63$ $(\mathrm{m}, 2 \mathrm{H}) ; 1,25(\mathrm{~m}, 18 \mathrm{H}) ; 0,88(\mathrm{t}, J=6,6 \mathrm{~Hz}, 3 \mathrm{H}) . \mathrm{RMN}{ }^{13} \mathrm{C}(75 \mathrm{MHz}$, $\left.\mathrm{CDCl}_{3}\right) \delta 171,87 ; 132,90 ; 125,22 ; 71,29 ; 46,53 ; 45,50 ; 36,66 ; 35,19$; 34,$64 ; 31,70 ; 29,42,29,27,29,22 ; 29,13 ; 28,96 ; 27,21 ; 25,95 ; 25,57$; 24,$74 ; 22,47 ; 18,20 ; 13,98$.

\section{N-Piperidinil-hexadecanamida (10a)}

$\mathrm{C}_{21} \mathrm{H}_{41}$ NO. Rendimento: 75\%. Aspecto: sólido branco, p.f.: 36-39 ${ }^{\circ}$ C. CCD: Rf = 0,5; AcOEt:Hex (3:7). IV (difusa): $\mathrm{cm}^{-1}$ (intensidade) 2922 (F); 2852 (F); 1647 (F); 1436 (f); 1253 (f); 852 (f). RMN ${ }^{1} \mathrm{H}$ $\left(300 \mathrm{MHz}, \mathrm{CDCl}_{3}\right) \delta 3,48(\mathrm{~m}, 4 \mathrm{H}) ; 2,37$ (t, $\left.J=7,8 \mathrm{~Hz}, 2 \mathrm{H}\right) ; 1,65-1,55$ 
$(\mathrm{m}, 8 \mathrm{H}) ; 1,25(\mathrm{~m}, 24 \mathrm{H}) ; 0,88(\mathrm{t}, J=6,9 \mathrm{~Hz}, 3 \mathrm{H}) . \mathrm{RMN}{ }^{13} \mathrm{C}(75 \mathrm{MHz}$, $\left.\mathrm{CDCl}_{3}\right) \delta 171,70 ; 46,50 ; 42,50 ; 33,46 ; 31,93 ; 29,69(3 \mathrm{C}) ; 29,67$ (3C); 29,$63 ; 29,56 ; 29,54 ; 29,46 ; 29,37 ; 26,12 ; 25,56 ; 24,58 ; 22,70 ; 14,13$.

\section{N-Piperidinil-octadecanamida (10b)}

$\mathrm{C}_{23} \mathrm{H}_{45} \mathrm{NO}$. Rendimento: $44 \%$. Aspecto: sólido branco, p.f.: 34-35 ${ }^{\circ} \mathrm{C}$. CCD: Rf = 0,5; AcOEt:Hex (3:7). IV (difusa) $\mathrm{cm}^{-1}$ (intensidade) 2922 (F); 1651 (F); 1440 (m); 1253 (m); 1138 (f); 1014 (f). RMN ${ }^{1} \mathrm{H}\left(300 \mathrm{MHz}, \mathrm{CDCl}_{3}\right) \delta 3,47(\mathrm{~m}, 2 \mathrm{H}) ; 3,32(\mathrm{~m}, 2 \mathrm{H}) ; 2,24(\mathrm{t}, J=7,9$ $\mathrm{Hz}, 2 \mathrm{H}) ; 1,55(\mathrm{~m}, 6 \mathrm{H}) ; 1,48(\mathrm{~m}, 2 \mathrm{H}) ; 1,18-1,28(\mathrm{~m}, 28 \mathrm{H}) ; 0,80(\mathrm{t}, J$ $=6,0 \mathrm{~Hz}, 3 \mathrm{H}) . \mathrm{RMN}{ }^{13} \mathrm{C}\left(75 \mathrm{MHz}, \mathrm{CDCl}_{3}\right) \delta 171,58 ; 46,66 ; 42,53$, 33,$38 ; 31,82 ; 29,59 ; 29,56 ; 29,52 ; 29,43 ; 29,39$ (2C); 29,34 (2C); 29,26 (2C); 29,22; 29,09; 26,47; 25,48; 25,42; 24,47; 22,58; $14,01$.

\section{N-Piperidinil-9-Z-octadecenamida (10c)}

$\mathrm{C}_{23} \mathrm{H}_{43} \mathrm{NO}$. Rendimento: $72 \%$. Aspecto: líquido amarelo. CCD: $\mathrm{Rf}=0,5 ; \mathrm{AcOEt}: \mathrm{Hex}(3: 7) . \mathrm{IV}\left(\mathrm{NaCl}\right.$, filme) $\mathrm{cm}^{-1}$ (intensidade) 3003 (F); 2924 (F); 2854 (F); 1629 (F); 1444 (F); 1217 (m); 1020 (f); $754(\mathrm{~m}) . \mathrm{RMN}^{1} \mathrm{H}\left(300 \mathrm{MHz}, \mathrm{CDCl}_{3}\right) \delta 5,34(\mathrm{~m}, 2 \mathrm{H}) ; 3,47(\mathrm{~m}$, $4 \mathrm{H}) ; 2,23$ (t, $J=7,8 \mathrm{~Hz}, 2 \mathrm{H}) ; 1,99(\mathrm{~m}, 4 \mathrm{H}) ; 1,63(\mathrm{~m}, 6 \mathrm{H}) ; 1,54(\mathrm{~m}$, 2H) $1,31(\mathrm{~m}, 20 \mathrm{H}) ; 0,88(\mathrm{t}, J=6,6 \mathrm{~Hz}, 3 \mathrm{H}) . \mathrm{RMN}{ }^{13} \mathrm{C}(75 \mathrm{MHz}$, $\left.\mathrm{CDCl}_{3}\right) \delta 171,69 ; 129,76(2 \mathrm{C}) ; 46,00,42,00 ; 33,38 ; 32,58 ; 31,88$ (3C); 29,75; 29,70; 29,50; 29,32; 29,30; 29,14; 27,16; 26,05; 25,51; 24,54 (2C); 22,66; 14,09.

\section{N-Piperidinil-12-hidróxi 9-Z-octadecenamida (10d)}

$\mathrm{C}_{23} \mathrm{H}_{43} \mathrm{NO}_{2}$. Rendimento: $52 \%$. Aspecto: líquido amarelo. CCD: $\mathrm{Rf}=0,3 ;$ AcOEt:Hex (3:7). IV ( NaCl, filme): $\mathrm{cm}^{-1}$ (intensidade) 3412 (F); 2926 (F), 2856 (F); 1627 (F); 1448 (F); 1342 (m); 1226 (m); 1041 (f); 860 (f); 752 (f). $\mathrm{RMN}^{1} \mathrm{H}\left(300 \mathrm{MHz}, \mathrm{CDCl}_{3}\right) \delta 5,49(\mathrm{~m}, 1 \mathrm{H}) ; 5,31$ $(\mathrm{m}, 1 \mathrm{H}) ; 3,53(\mathrm{~m}, 1 \mathrm{H}) ; 3,47(\mathrm{~m}, 4 \mathrm{H}) ; 3,32(\mathrm{~m}, 1 \mathrm{H}) ; 2,23(\mathrm{t}, J=7,5$ $\mathrm{Hz}, 2 \mathrm{H}) ; 1,96(\mathrm{~m}, 4 \mathrm{H}) ; 1,55(\mathrm{~m}, 8 \mathrm{H}) ; 1,48(\mathrm{~m}, 2 \mathrm{H}) ; 1,24(\mathrm{~m}, 16 \mathrm{H})$; $0,81(\mathrm{~m}, 3 \mathrm{H}) . \mathrm{RMN}{ }^{13} \mathrm{C}\left(75 \mathrm{MHz}, \mathrm{CDCl}_{3}\right) \delta 171,51 ; 133,22 ; 125,19$; 71,$43 ; 46,67 ; 42,54 ; 36,77 ; 35,29 ; 33,90 ; 31,78 ; 29,63 ; 29,51 ; 29,39$; 29,$29 ; 29,22 ; 29,05 ; 27,30 ; 26,51 ; 25,66 ; 25,52 ; 25,38 ; 24,51 ; 14,03$.

\section{N-Morfolinil-hexadecanamida (11a)}

$\mathrm{C}_{20} \mathrm{H}_{39} \mathrm{NO}_{2}$. Rendimento: 75\%. Aspecto: sólido amarelo, p.f.: 38$40{ }^{\circ} \mathrm{C}$. CCD: Rf=0,5; AcOEt:Hex (3:7). IV (difusa) $\mathrm{cm}^{-1}$ (intensidade) 2918 (F); 2848 (F); 1641 (F); 1469 (m); 1265 (m); 1111 (F); 962 (f). $\mathrm{RMN}{ }^{1} \mathrm{H}\left(300 \mathrm{MHz}, \mathrm{CDCl}_{3}\right) \delta 3,60(\mathrm{~m}, 6 \mathrm{H}) ; 3,38(\mathrm{~m}, 2 \mathrm{H}) ; 2,23(\mathrm{t}$, $J=7,5 \mathrm{~Hz}, 2 \mathrm{H}) ; 1,65(\mathrm{~m}, 2 \mathrm{H}) ; 1,55(\mathrm{~m}, 2 \mathrm{H}) ; 1,18(\mathrm{~m}, 22 \mathrm{H}) ; 0,81(\mathrm{t}$, $J=6,3 \mathrm{~Hz}, 3 \mathrm{H}) . \mathrm{RMN}{ }^{13} \mathrm{C}\left(75 \mathrm{MHz}, \mathrm{CDCl}_{3}\right) \delta 171,95 ; 66,93(2 \mathrm{C})$; 46,$01 ; 41,80 ; 33,12 ; 31,88 ; 29,64$ (3C); 29,61 (2C); 29,58; 29,47; 29,$44 ; 29,39 ; 29 ; 32 ; 25,23 ; 22,65 ; 14$

\section{N-Morfolinil-octadecanamida $(\mathbf{1 1 b})$}

$\mathrm{C}_{22} \mathrm{H}_{43} \mathrm{NO}_{2}$. Rendimento: $59 \%$. Aspecto: sólido pastoso amarelopalha. CCD: Rf=0,5; AcOEt:Hex (3:7). IV (difusa) $\mathrm{cm}^{-1}$ (intensidade) 2914 (F); 2848 (F); 1747 (m); 1641 (F); 1469 (F); 1111 (F); 854 (f); 717 (m). RMN ${ }^{1} \mathrm{H}\left(300 \mathrm{MHz}, \mathrm{CDCl}_{3}\right) \delta 3,59(\mathrm{~m}, 6 \mathrm{H}) ; 3,40(\mathrm{~m}, 2 \mathrm{H})$; $2,25(\mathrm{t}, J=7,8 \mathrm{~Hz}, 2 \mathrm{H}) ; 1,57(\mathrm{~m}, 2 \mathrm{H}) ; 1,29(\mathrm{~m}, 28 \mathrm{H}) ; 0,80(\mathrm{t}, J=$ $6,9 \mathrm{~Hz}, 3 \mathrm{H}) . \mathrm{RMN}{ }^{13} \mathrm{C}\left(75 \mathrm{MHz}, \mathrm{CDCl}_{3}\right) \delta 172,29 ; 67,06(2 \mathrm{C}) ; 44,3$; 42,00; 34,17; 32,17; 29,94 (4C); 29,91 (3C); 29,86; 29,76; 29,72; 29,$67 ; 29,61 ; 25,53 ; 22,94 ; 14,37$.

\section{N-Morfolinil-9-Z-octadecenamida (11c)}

$\mathrm{C}_{22} \mathrm{H}_{41} \mathrm{NO}_{2}$. Rendimento: $57 \%$. Aspecto: líquido amarelo-palha. CCD: Rf= 0,5; AcOEt:Hex (3:7). IV (difusa) $\mathrm{cm}^{-1}$ (intensidade) 2922 (F); 2850 (F); 1728 (F); 1643 (F); 1454 (F); 1271 (m); 1116 (F); 1033 (f); 850 (f). RMN ${ }^{1} \mathrm{H}\left(300 \mathrm{MHz}, \mathrm{CDCl}_{3}\right) \delta 5,24(\mathrm{~m}, 2 \mathrm{H}) ; 3,57(\mathrm{~m}$, $6 \mathrm{H}) ; 3,36(\mathrm{~m}, 2 \mathrm{H}) ; 2,21(\mathrm{t}, J=7,5 \mathrm{~Hz}, 2 \mathrm{H}) ; 1,92(\mathrm{~m}, 4 \mathrm{H}) ; 1,52(\mathrm{~m}$, $2 \mathrm{H}) ; 1,17(\mathrm{~m}, 20 \mathrm{H}) ; 0,80(\mathrm{t}, J=7,2 \mathrm{~Hz}, 3 \mathrm{H}) . \mathrm{RMN}{ }^{13} \mathrm{C}(75 \mathrm{MHz}$,
$\left.\mathrm{CDCl}_{3}\right) \delta 171,85 ; 129,86(2 \mathrm{C}) ; 66,80 ; 66,53 ; 45,93 ; 41,75 ; 32,98$; 31,$77 ; 29,62 ; 29,56 ; 29,39 ; 29,29 ; 29,18$ (2C); 29,09; 29,00; 27,07 (2C); 25, 12; 22,55; 13,99.

\section{N-Morfolinil-12-hidróxi-9-Z-octadecenamida (11d)}

$\mathrm{C}_{22} \mathrm{H}_{41} \mathrm{NO}_{3}$. Rendimento: $66 \%$. Aspecto: sólido pastoso amarelopalha, CCD: Rf=0,5; AcOEt:Hex (3:7). IV (difusa) $\mathrm{cm}^{-1}$ (intensidade) $3441(\mathrm{~F}) ; 2926(\mathrm{~F}) ; 2854(\mathrm{~F}) ; 1624(\mathrm{~F}) ; 1433(\mathrm{~F}) ; 1271(\mathrm{~m}) ; 1116$ (F); 1035 (f); 850 (f). RMN ${ }^{1} \mathrm{H}\left(300 \mathrm{MHz}, \mathrm{CDCl}_{3}\right) \delta 5,49$ (m, 1H); $5,37(\mathrm{~m}, 1 \mathrm{H}) ; 3,60(\mathrm{~m}, 6 \mathrm{H}) ; 3,39(\mathrm{~m}, 2 \mathrm{H}) ; 2,23(\mathrm{t}, J=7,5 \mathrm{~Hz}, 2 \mathrm{H})$; 2,13 (m, 2H); 1,96 (m, 2H); 1,55 (m, 2H); 1,21 (m, 20H); 0,81 (t, J $=7,2 \mathrm{~Hz}, 3 \mathrm{H}) . \mathrm{RMN}{ }^{13} \mathrm{C}\left(75 \mathrm{MHz}, \mathrm{CDCl}_{3}\right) \delta 172,17 ; 133,49 ; 125,51$; 71,$68 ; 67,19 ; 66,92 ; 46,29 ; 42,12 ; 37,07 ; 35,59 ; 33,35 ; 32,09 ; 29,82$; 29,62 (2C); 29,50;29,35; 27,61; 25,97; 25,45; 22,87; 14,35.

\section{$N$-(2-Hidroxietil) hexadecanamida (12a)}

$\mathrm{C}_{18} \mathrm{H}_{37} \mathrm{NO}_{2}$. Rendimento: $62 \%$. Aspecto: sólido branco, p.f.: $34-35$ ${ }^{\circ} \mathrm{C}, \mathrm{CCD}: \mathrm{Rf}=0,4 ; \mathrm{CHCl}_{3}: \mathrm{MeOH}(97: 3)$. IV (difusa) $\mathrm{cm}^{-1}$ (intensidade) $3292(\mathrm{~F}) ; 2920(\mathrm{~F}) ; 2850(\mathrm{~F}) ; 1643(\mathrm{~F}) ; 1552(\mathrm{~m}) ; 1522(\mathrm{~m})$; 1465 (f); 1056 (f); 721 (f). RMN ${ }^{1} \mathrm{H}\left(300 \mathrm{MHz}, \mathrm{CDCl}_{3}\right) \delta 6,38$ (sl, $1 \mathrm{H}) ; 3,72(\mathrm{~m}, 2 \mathrm{H}) ; 3,40(\mathrm{~m}, 3 \mathrm{H}) ; 2,22(\mathrm{t}, J=7,2 \mathrm{~Hz}, 2 \mathrm{H}) ; 1,61(\mathrm{~m}$, $2 \mathrm{H}), 1,25(\mathrm{~m}, 24 \mathrm{H}) ; 0,88(\mathrm{t}, J=6,9 \mathrm{~Hz}, 3 \mathrm{H}) . \mathrm{RMN}{ }^{13} \mathrm{C}(75 \mathrm{MHz}$, $\left.\mathrm{CDCl}_{3}\right) \delta 174,72 ; 62,21 ; 42,33,36,63 ; 33,85 ; 31,88 ; 29,72 ; 29,65$; 29,$62 ; 29,59 ; 29,47 ; 29,32 ; 29,26 ; 29,23 ; 27,18 ; 25,71 ; 22,65 ; 14,08$.

\section{$N$-(2-Hidroxietil) octadecanamida (12b)}

$\mathrm{C}_{20} \mathrm{H}_{41} \mathrm{NO}_{2}$. Rendimento: $88 \%$. Aspecto: sólido pastoso amarelopalha. $\mathrm{CCD}: \mathrm{Rf}=0,4 ; \mathrm{CHCl}_{3}: \mathrm{MeOH}(97: 3)$. IV (difusa) $\mathrm{cm}^{-1}$ (intensidade) 3294 (F); 3091 (f); 2916 (F); 2848 (F); 1641 (F); 1558 (m); 1465 (m); 1361 (f); 1047 (f); 721 (f). $\mathrm{RMN}^{1} \mathrm{H}\left(300 \mathrm{MHz}, \mathrm{CDCl}_{3}\right) \delta$ $6,13(\mathrm{sl}, 1 \mathrm{H}) ; 3,66(\mathrm{~m}, 2 \mathrm{H}) ; 3,40(\mathrm{~m}, 3 \mathrm{H}) ; 2,13(\mathrm{t}, J=7,5 \mathrm{~Hz}, 2 \mathrm{H})$; $1,57(\mathrm{~m}, 2 \mathrm{H}) ; 1,36(\mathrm{~m}, 28 \mathrm{H}) ; 0,83(\mathrm{t}, J=6,5 \mathrm{~Hz}, 3 \mathrm{H}) . \mathrm{RMN}{ }^{13} \mathrm{C}(75$ $\left.\mathrm{MHz}, \mathrm{CDCl}_{3}\right) \delta 174,98 ; 62,55 ; 42,66 ; 36,92 ; 32,16 ; 29,94$ (6C); 29,90; 29,$87 ; 29,74 ; 29,60 ; 29,53 ; 25,99 ; 23,36 ; 22,93 ; 14,36$.

\section{$N$-(2-Hidroxietil)9-Z-octadecenamida (12c)}

$\mathrm{C}_{20} \mathrm{H}_{39} \mathrm{NO}_{2}$. Rendimento: $66 \%$. Aspecto: sólido amarelo-palha, p.f.: $55-56{ }^{\circ} \mathrm{C}$. CCD: $\mathrm{Rf}=0,4 ; \mathrm{CHCl}_{3}: \mathrm{MeOH}(97: 3)$. IV (difusa) $\mathrm{cm}^{-1}$ (intensidade) 3296 (F), 3089 (f); 2920 (F), 2850 (F), 1643 (F); 1565 (m); 1465 (m); 1381 (f); 1265 (f); 1211 (f); 1122 (f); 1058 (m); 1037 (f), 721 (f). RMN ${ }^{1} \mathrm{H}\left(300 \mathrm{MHz}, \mathrm{CDCl}_{3}\right) \delta 6,10(\mathrm{sl}, 1 \mathrm{H}) ; 5,34(\mathrm{~m}$, $2 \mathrm{H}) ; 3,72(\mathrm{~m}, 2 \mathrm{H}) ; 3,41(\mathrm{~m}, 3 \mathrm{H}) ; 2,23(\mathrm{t}, J=7,2 \mathrm{~Hz}, 2 \mathrm{H}) ; 2,01(\mathrm{~m}$, $4 \mathrm{H}) ; 1,63(\mathrm{~m}, 2 \mathrm{H}) ; 1,30(\mathrm{~m}, 20 \mathrm{H}) ; 0,88(\mathrm{t}, J=6,9 \mathrm{~Hz}, 3 \mathrm{H})$. RMN ${ }^{13} \mathrm{C}\left(75 \mathrm{MHz}, \mathrm{CDCl}_{3}\right) \delta 174,61 ; 129,98(2 \mathrm{C}) ; 62,26 ; 42,39 ; 36,63$; 31,$86 ; 29,73 ; 29,68 ; 29,62 ; 29,49 ; 29,33 ; 29,28 ; 29,24 ; 29,11 ; 27,19$; 27,$14 ; 25,71 ; 22,64 ; 14,08$.

\section{$\mathrm{N}$-(2-hidroxietil)12- hidróxi-9-Z-octadecenamida (12d)}

$\mathrm{C}_{20} \mathrm{H}_{39} \mathrm{NO}_{3}$. Rendimento: 53\%. Aspecto: sólido amarelo, p.f.: 47-50 ${ }^{\circ} \mathrm{C}$. CCD: $\mathrm{Rf}=0,4 ; \mathrm{CHCl}_{3}: \mathrm{MeOH}$ (97:3). IV (difusa) $\mathrm{cm}^{-1}$ (intensidade) 4330 (f); 3294 (F); 2922 (F); 2850 (F); 1643 (F); 1562 (m); 1465 (m); 1211 (f); 1058 (m); 856 (f). RMN ${ }^{1} \mathrm{H}\left(300 \mathrm{MHz}, \mathrm{CDCl}_{3}\right.$ ) $\delta 6,63(\mathrm{sl}, 1 \mathrm{H}) ; 5,52(\mathrm{~m}, 1 \mathrm{H}) ; 5,35(\mathrm{~m}, 1 \mathrm{H}) ; 3,68(\mathrm{~m}, 3 \mathrm{H}) ; 3,39(\mathrm{~m}$, $4 \mathrm{H}) ; 2,20(\mathrm{~m}, 4 \mathrm{H}) ; 2,05(\mathrm{~m}, 2 \mathrm{H}) ; 1,61(\mathrm{~m}, 2 \mathrm{H}) ; 1,45(\mathrm{~m}, 2 \mathrm{H}) ; 1,30(\mathrm{~m}$, $16 \mathrm{H}) ; 0,88$ (t, $J=7,0 \mathrm{~Hz}, 3 \mathrm{H}) . \mathrm{RMN}{ }^{13} \mathrm{C}\left(75 \mathrm{MHz}, \mathrm{CDCl}_{3}\right) \delta 174,62$; 132,$97 ; 125,25 ; 71,47 ; 61,77 ; 42,19 ; 36,70 ; 36,46 ; 35,21 ; 31,75$; 29,$61 ; 29,43 ; 29,27 ; 29,04 ; 28,91 ; 27,21 ; 25,63 ; 25,59 ; 22,53 ; 14,01$.

\section{$N$-Benzil-hexadecanamida (13a)}

$\mathrm{C}_{23} \mathrm{H}_{39} \mathrm{NO}$. Rendimento: $65 \%$. Aspecto: sólido pastoso branco, p.f.: $94-96{ }^{\circ} \mathrm{C}$. CCD: $\mathrm{Rf}=0,5$; AcOEt:Hex (3:7). IV (difusa) $\mathrm{cm}^{-1}$ (intensidade) 3294 (F); 2916 (F); 2848 (F); 1631 (F); 1550 (F); 1456 (m); 1355 (f); 1116 (f); 725 (f). $\mathrm{RMN}^{1} \mathrm{H}\left(300 \mathrm{MHz}, \mathrm{CDCl}_{3}\right) \delta 7,32$ 
$(\mathrm{m}, 5 \mathrm{H}) ; 5,82(\mathrm{sl}, 1 \mathrm{H}) ; 4,44(\mathrm{~d}, J=5,7 \mathrm{~Hz}, 2 \mathrm{H}) ; 2,20(\mathrm{t}, J=7,8 \mathrm{~Hz}$, $2 \mathrm{H}) ; 1,66(\mathrm{~m}, 2 \mathrm{H}) ; 1,25(\mathrm{~m}, 24 \mathrm{H}) ; 0,90(\mathrm{t}, J=6,9 \mathrm{~Hz}, 3 \mathrm{H}) . \mathrm{RMN}$ ${ }^{13} \mathrm{C}\left(75 \mathrm{MHz}, \mathrm{CDCl}_{3}\right) \delta 173,08 ; 138,34 ; 128,66(2 \mathrm{C}) ; 127,78(2 \mathrm{C})$; 127,$46 ; 43,57 ; 36,74 ; 31,90 ; 29,67$ (4C); 29,63 (2C); 29,58; 29,47; 29,$33 ; 29,29 ; 25,77 ; 22,66 ; 14,09$. GC-MS tempo de retenção: 14,9 min., $m / z$. 91 (53\%); 106 (27\%); 149 (100\%); $162(20 \%) ; 345\left(\mathrm{M}^{+}\right)$.

\section{$N$-Benzil-octadecanamida $(\mathbf{1 3 b})$}

$\mathrm{C}_{25} \mathrm{H}_{43} \mathrm{NO}$. Rendimento: $60 \%$. Aspecto: sólido pastoso branco, p.f.: $93-95^{\circ} \mathrm{C}$. CCD: $\mathrm{Rf}=0,5 ; \mathrm{CHCl}_{3}: \mathrm{MeOH}(97: 3)$. IV (difusa) $\mathrm{cm}^{-1}$ (intensidade) 3305 (F); 2951 (F); 2360 (m); 2341 (f); 1637 (F); 1548 (m); 1454 (f); 1355 (f); 729 (f). $\mathrm{RMN}^{1} \mathrm{H}\left(300 \mathrm{MHz}, \mathrm{CDCl}_{3}\right) \delta 7,35$ $(\mathrm{m}, 5 \mathrm{H}) ; 5,78(\mathrm{sl}, 1 \mathrm{H}) ; 4,44(\mathrm{~d}, J=5,7 \mathrm{~Hz}, 2 \mathrm{H}) ; 2,20(\mathrm{t}, J=7,8 \mathrm{~Hz}$, $2 \mathrm{H}) ; 1,64(\mathrm{~m}, 2 \mathrm{H}) ; 1,25(\mathrm{~m}, 28 \mathrm{H}) ; 0,88$ (t, $J=6,6 \mathrm{~Hz}, 3 \mathrm{H})$. RMN ${ }^{13} \mathrm{C}\left(75 \mathrm{MHz}, \mathrm{CDCl}_{3}\right) \delta 172,99 ; 138,40 ; 128,67$ (2C); 127,80 (2C); 127,46; 43,56; 36,80; 31,90; 29,67 (5C); 29,63 (3C); 29,59; 29,48; 29,$34 ; 29,30 ; 25,75 ; 22,67 ; 14,10$. GC-MS tempo de retenção: 16,6 min., $m / z 91$ (57\%); 106 (25\%); 149 (100\%); $162(23 \%) ; 373\left(\mathrm{M}^{+}\right)$;

\section{$N$-Benzil-9-Z-octadecenamida (13c)}

$\mathrm{C}_{25} \mathrm{H}_{41} \mathrm{NO}$. Rendimento: $61 \%$. Aspecto: sólido pastoso amarelopalha. CCD: Rf= 0,5; AcOEt:Hex (3:7) (7:3). IV (difusa) $\mathrm{cm}^{-1}$ (intensidade) $3298(\mathrm{~F}) ; 2924(\mathrm{~F}) ; 2852(\mathrm{~F}) ; 1647(\mathrm{~F}) ; 1552(\mathrm{~F}) ; 1452$ (F); 1255 (m); 1028 (f); 698 (m). RMN ${ }^{1} \mathrm{H}\left(300 \mathrm{MHz}, \mathrm{CDCl}_{3}\right) \delta 7,31$ (m, 5H); 6,00 (sl, 1H); 5,34 (m, 2H); 4,41 (d, $J=5,7 \mathrm{~Hz}, 2 \mathrm{H}) ; 2,18$ (t, $J=7,8 \mathrm{~Hz}, 2 \mathrm{H}) ; 2,00(\mathrm{~m}, 4 \mathrm{H}) ; 1,63(\mathrm{~m}, 2 \mathrm{H}) ; 1,26(\mathrm{~m}, 20 \mathrm{H}) ; 0,87$ (t, $J=6,9 \mathrm{~Hz}, 3 \mathrm{H}) . \mathrm{RMN}{ }^{13} \mathrm{C}\left(75 \mathrm{MHz}, \mathrm{CDCl}_{3}\right) \delta 173,07 ; 138,40$; 129,$92 ; 129.67 ; 128,58$ (2C); 127,70 (2C); 127, 36; 43,45; 36,67; 31,$84 ; 29,70 ; 29,64 ; 29,59 ; 29,46 ; 29,29 ; 29,26 ; 29,23 ; 29,20 ; 27,15$; 27,$11 ; 25,71 ; 22,62 ; 14,06$

\section{N-Benzil-12-hidróxi-9-Z-octadecenamida (13d)}

$\mathrm{C}_{25} \mathrm{H}_{41} \mathrm{NO}_{2}$. Rendimento: $63 \%$. Aspecto: sólido pastoso amarelo. CCD: Rf= 0,5; AcOEt:Hex (3:7). IV (difusa) $\mathrm{cm}^{-1}$ (intensidade) 3305 (F); 2924 (F); 2848 (F); 1639 (F); 1548 (F); 1454 (F); 1259 (f); 1078 (f); $696(\mathrm{~m}) . \mathrm{RMN}^{1} \mathrm{H}\left(300 \mathrm{MHz}, \mathrm{CDCl}_{3}\right) \delta 7,31(\mathrm{~m}, 5 \mathrm{H}) ; 5,83(\mathrm{sl}$, 1H); 5,57 (m, 1H); 5,41 (m, 1H); 4,45 (d, $J=5,4 \mathrm{~Hz}, 2 \mathrm{H}) ; 3,62(\mathrm{~m}$, $1 \mathrm{H}) ; 2,21(\mathrm{~m}, 4 \mathrm{H}) ; 2,03(\mathrm{~m}, 2 \mathrm{H}) ; 1,79(\mathrm{~m}, 1 \mathrm{H}) ; 1,65(\mathrm{~m}, 2 \mathrm{H}) ; 1,46$ $(\mathrm{m}, 2 \mathrm{H}) ; 1,30(\mathrm{~m}, 16 \mathrm{H}) ; 0,88(\mathrm{t}, J=6,9 \mathrm{~Hz}, 3 \mathrm{H}) . \mathrm{RMN}{ }^{13} \mathrm{C}(75 \mathrm{MHz}$, $\left.\mathrm{CDCl}_{3}\right) \delta 173,05 ; 138,42 ; 133,23 ; 128,64$ (2C); 127,77 (2C); 127,42; 125,$26 ; 71,46 ; 43,52 ; 36,81 ; 35,33 ; 31,81 ; 29,51 ; 29,32$ (2C); 29, 18 ; 29,$12 ; 29,03 ; 27,30(2 \mathrm{C}) ; 25,70 ; 22,60 ; 14,06$.

\section{AGRADECIMENTOS}

À CAPES e ao CNPq, pelas bolsas de mestrado e auxílio financeiro concedido. À Prof ${ }^{\text {a. }}$ G. dos S. Trindade, Instituto de Ciências Biológicas, FURG e aos Profs. D. Russowsky, R. G. da Rosa, A. A. Merlo e P. H. Schneider do Instituto de Química, UFRGS, pelas análises de RMN.

\section{REFERÊNCIAS}

1. Bezuglov, V. V.; Brobov, M. Y.; Archakov, A. V.; Biochemistry (Moscow) 1998, 63, 22.

2. Farrel, E. K.; Merkler, D. J.; Drug Discover Today 2008, 13, 558.

3. Gee, A. J.; Groen, L. A.; Johnson, M. E.; J. Chromatogr., A 1999, 849, 541.

4. Bezuglov, V.; Brobov, M.; Gretskaya, N.; Gonchar, A.; Zinchenko, G.; Melck, D.; Bisogno, T.; Di Marzo, V.; Kuklev, D.; Rossi, J. C.; Vidal, J. P.; Durand, T.; Bioorg. Med. Chem. Lett. 2001, 11, 447.

5. Levinson, W. E.; Kuo, T. M..; Knothe, G.; Bioresour. Technol. 2008, 99, 2706.
6. Urbani, P.; Cavallo, P.; Cascio, M. G.; Buonerba, M.; De Martino, G.; Di Marzo, V.; Saturnino, C.; Bioorg. Med. Chem. Lett. 2006, 16, 138.

7. Dembitsky, V. M.; Shkrob, I.; Rozentsvet, O. A.; Phytochemistry 2000, $54,965$.

8. Kawasaki, W.; Matsui, K; Akakabe, Y.; Itai, N.; Kajiwar, T.; Phytochemistry 1998, 47, 27.

9. Wang, L.; An, X.; Yang, F.; Xin, Z.; Zhao, L.; Hu, Q.; Food Chem. 2008, $108,616$.

10. Zhao, J.; Muhammad, I.; Dunbar, D. C.; Mustafa, J.; Khan, I. A.; J. Agric. Food Chem. 2005, 53, 690.

11. Kashiwada, Y.; Ito, C.; Katagiri, H.; Mase, I.; Komatsu, K.; Namba, T.; Ikeshiro, Y.; Phytochemistry 1997, 44, 1125.

12. Mansoor, T. A.; Shinde, P. B.; Luo, X.; Hong, J.; Lee, C. O.; Sim, C. J.; Son, B. W; Jung, J. H.; J. Nat. Prod. 2007, 70, 1481.

13. Akanmu, M. A.; Adeosun, S. O.; Ilesanmi O. R.; Behav. Brain Res. 2007, 182,88 .

14. Guan, X.; Cravatt, B. F.; Ehring, G. R.; Hall, J. E.; Boger, D. L.; Lerner, R. A.; Gilula, N. B.; J. Cell Biol. 1997, 139, 1785.

15. Huitrón-Reséndiz, S.; Gombart, L.; Cravatt, B. F.; Henriksen, S. J.; Exp. Neurol. 2001, 172, 235.

16. Arafat, E. S.; Trimble, J. W.; Andersen, R. N.; Dass, C.; Desiderio, D. M.; Life Sci. 1989, 45, 1679.

17. Spoto, B.; Fezza, F.; Parlongo, G.; Battista, N.; Sgro, E.; Gasperi, V.; Zoccali, C.; Macarrone, M.; Biochimie 2006, 88, 1889.

18. Deutsch, D. G.; Chin, S. A.; Biochem. Pharmacol. 1993, 46, 791.

19. Hillard, C. J.; Wilkinson D. M.; Edgemond, W. S.; Campbell, W. B.; Biochim. Biophys. Acta 1995, 1257, 249.

20. Fu, J.; Astarita, G.; Gaetani, S.; Kim, J.; Cravatt, B. F.; Mackie, K.; Piomelli, D.; J. Biol. Chem. 2007, 282, 1518.

21. Watanabe, K.; Kayano, Y.; Matsunaga, T.; Yamamoto, I.; Yoshimura, H.; Biol. Pharm. Bull. 1996, 19, 1109.

22. Ueda, N.; Kurahashi, Y.; Yamamoto, S.; Tokonuga, T.; J. Biol. Chem. 1995, 270, 23823.

23. Madl, T.; Mittelbach, M.; Analyst 2005, 130, 565.

24. Amarant, T.; Vered, Y.; Bohak, Z.; Biotechnol. Appl. Biochem. 1989, 11, 49.

25. Kwon, H. J.; Hwang, S. E.; Han, J. T.; Kim, C. J.; Rho, J. R.; Shin, J.; J. Microbiol. Biotechnol. 2001, 11, 1018 .

26. Bisogno, T.; Katayama, K.; Melck, D.; Ueda, N.; De Petrocellis, L.; Yamamoto, S.; Di Marzo, V.; Eur. J. Biochem. 1998, 254, 634

27. Virolleaud, M. A.; Menant, C.; Fenet, B.; Piva, O.; Tetrahedron Lett. 2006, 47, 5127.

28. Sitachitta, N.; Gerwick, W. H.; J. Nat. Prod. 1998, 61, 681.

29. Davies, I. R.; Cheeseman, M.; Niyadurupola, D. G.; Bull, S. D.; Tetrahedron Lett. 2005, 46, 5547.

30. Tan, L. T.; Chang, Y. Y.; Ashootosh, T.; Phytochemistry 2008, 69, 2067.

31. Re, G.; Barbero, R.; Miolo, A.; Di Marzo, V.; The Veterinary J. 2007, $173,21$.

32. Berdyshev, E. V.; Boichot, E.; Lagente, V.; J. Lipid Mediators Cell Signalling 1996, 15, 49.

33. Driscoll, W. J.; Chaturvedi, S.; Mueller, G. P.; J. Biol. Chem. 2007, 282, 22353.

34. Aviello, G.; Matias, I.; Capasso, R.; Petrosino, S.; Borrelli, F.; Orlando, P.; Romano, B.; Capasso, F.; Di Marzo, V.; Izzo, A. A.; J. Mol. Med. 2008, 86, 413.

35. Benz, G. Em Comprehensive Organic Synthesis: selectivity, strategy and efficiency in modern organic chemistry; Trost, B. M.; Fleming, I., eds.; Pergamon Press: Oxford, New York, Seoul, Tokyo, 1991, cap. 2.3, vol. 6, p. 381.

36. Guo, Z.; Dowdy, E. D.; Li, W. S.; Polniaszek, R.; Delaney, E.; Tetrahedron Lett. 2001, 42, 1843.

37. Serkov, I. V.; Bezuglov, V. V.; Chem. Nat. Compd. 2007, 43, 103.

38. Montalbetti, C. A. G. N.; Falque, V.; Tetrahedron 2005, 61, 10827. 
39. Ech-Chahad, A.; Minassi, A.; Berton, L.; Appendino, G.; Tetrahedron Lett. 2005, 46, 5113.

40. Ranger, C. M.; Winter, R. E. K.; Rottinghaus, G. E.; Backus, E. A.; Johnson, D. W.; Phytochemistry 2005, 66, 529.

41. Mazzari, S.; Canella, R.; Petrelli, L.; Marcolongo, G.; Leon, A.; Eur. J. Pharmacol. 1996, 300, 227.

42. Yao, F.; Li, C.; Vadivel, S. K.; Bowman, A. L.; Makriyannis, A.; Bioorg. Med. Chem. Lett. 2008, 18, 5912.

43. Hanuš, L. O.; Fales, H. M.; Spande, T. F.; Basile, A. S.; Anal. Biochem. 1999, 270, 159.

44. Fu, J.; Gaetani, S.; Oveisi, F.; Lo Verme, J.; Serrano, A.; Fonseca, F. R.; Rosengarth, A.; Luecke, H.; Di Giacomo, B.; Tarzia, G.; Piomelli, D.; Nature 2003, 425, 90.

45. Lakshminarayana, G.; Paulose, M. M.; Neeta-Kumari, B.; J. Am. Oil Chem. Soc. 1984, 61, 1871.

46. Turner, C.; Whitehand, L. C.; Nguyen, T.; Mackeon, T.; J. Agric. Food Chem. 2004, 52, 26.
47. Ahmad, S.; Ashraf, S. M.; Naqui, F.; Yadav, S.; Hasnat, A.; Prog. Org. Coat. 2004, 51, 250.

48. Lopes, C.; Domingues, B.; Rodrigues, M.; Clementin, R.; Trindade, G.; D’Oca, M. M.; Resumos do XVI Encontro de Química da Região Sul, Blumenau, Brasil, 2008.

49. Domingues, B.; Lopes, C.; Duarte, R.; Clementin, R.; D’Oca, M. M.; Trindade, G.; Resumos da XXIII Reunião Anual da Federação de Sociedade de Biologia Experimental - FeSBE, Águas de Lindóia, Brasil, 2008.

50. http://www.sigmaaldrich.com/brazil.html, acessada em Junho 2009.

51. Alcantara, R.; Amores, J.; Canoira, L.; Fidalgo, E.; Franco, M. J.; Navarro, A.; Biomass Bioenerg. 2000, 18, 515.

52. Haertel, P.; Dissertação de Mestrado, Universidade Federal de Rio Grande, Brasil, 2009.

53. Perin, G.; Álvaro, G.; Westphal, E.; Viana, L. H.; Jacob, R. G.; Lenardão, E. J.; D’Oca, M. G. M.; Fuel 2008, 87, 2838. 